Adhesi on st ate det ecti on by vi si on and its appl i cat i on to aut onat i c mi cro mani pul at i on

\begin{tabular}{|l|l|}
\hline 著者 & Wat nabe Tet suyou, Ser i t a Yut aka \\
\hline $\begin{array}{l}\text { j our nal or } \\
\text { publ i cat i on t i t I e }\end{array}$ & $\begin{array}{l}\text { Pr oceedi ngs of the I EEE/RSJ I nt er nat i onal } \\
\text { Conf er ence on I nt el I i gent Robot s and Syst ens } \\
\text { ( I ROS) }\end{array}$ \\
\hline vol une & 2008 \\
\hline number & 4650789 \\
\hline page r ange & $458-463$ \\
\hline year & 2008 01-01 \\
\hline URL & ht t p: //hdl . handl e. net /2297/35227 \\
\hline
\end{tabular}




\title{
Adhesion state detection by vision and its application to automatic micro manipulation
}

\author{
Tetsuyou Watanabe and Yutaka Serita
}

\begin{abstract}
In our previous paper, we proposed a method for reducing adhesion forces by oscillation. However, the following problems have not been still resolved: (1) Since adhesion state was checked by analyzing the data obtained by laser displacement meter, this method is available in only limited situations. (2) Automatic checking system for adhesion state was not developed. (3) Automatic micro manipulation system was not developed. Considering the above, in this paper, we propose a method to automatically check adhesion state by vision. Firstly, we develop a method to estimate the amplitude of the oscillation using the blur resulted from the oscillation. We call the estimated amplitude AIV (amplitude indicating value). Then, we develop a method for checking adhesion state by AIV. Based on the checking method, we develop a automatic micro manipulation system for pick and place operation. The validity of our method is shown by experiments.
\end{abstract}

\section{INTRODUCTION}

$\mathrm{R}$ ECENTLY, there has been a growing interest in a manipulation of a micro/nano sized object. It is the technology required to assemble or maintain microcomputers, micro electronics parts, a micro medical equipment, and so on. Different from a manipulation in a macro range, we cannot neglect an adhesion force between a micro object and an endeffector. In a macro range, the van der Waals, capillary, and electrostatic forces (proportional to surface area) become more significant than the inertial and gravitational forces (proportional to volume), because of a scale effect[1], [2]. These adhesion forces (van der Waals, capillary, and electrostatic forces) cause the adhesion between a micro object and an endeffector. Therefore, even a basic operation such as a pick and place operation is very hard to complete. Especially, a release of an object from an endeffector is very difficult.

In order to resolve the releasing problem, many methods have been developed, including the adhesion-type micro endeffector[3], the vacuum gripping tool[4], releasing by slopping and oscillating an endeffector[5]-[7], releasing by control of electrostatic force[8], and releasing strategy based on environment information[9]. However, the problems such as the hardness of control after the release[3]-[8], the hardness of practical use due to the harness of acquisition of all required information[9] were not resolved.

Recently, we developed a new strategy to cope with the adhesion force[10]. When bringing an oscillated endeffector

T. Watanabe and Y. Serita are with Graduate School of Natural Science \& Technology, Kanazawa University, Kanazawa, 920-1192, Japan (e-mail: te-watanabe@ieee.org). close to a micro object on a substrate and contacting the endeffector with the object, the adhesion force between the object and the endeffector becomes smaller than that between the object and the substrate. Then, it is easy to remove the endeffector from the object. Using this strategy, we can accurately manipulate micro object like macro manipulation.

But, this strategy is not always available. When the amount of pushing the object by endeffector is large, the oscillation becomes small, and then the adhesion force is not reduced enough. To investigate the phenomenon, laser displacement meter was used in the previous paper[10]. When lower mode frequencies (than the frequency of the input oscillation) are excited, adhesion force is reduced enough. When lower frequencies are not exited, adhesion force is not reduced enough. However, this method is available in only limited situations since the laser displacement meter must be fixed and can measure only limited area/point. If something blocks the light/laser or the target leaves measuring point, the laser displacement meter can not measure the target. Therefore, it is hard to check the adhesion state by this method in micro manipulation. Also, the method for automatically checking adhesion state was not developed. Applying FFT to the measured data by the laser displacement meter, we search and detect the lower mode frequencies manually. Since this method takes much time, it is not easy to check adhesion force on-line. Also, controlling method to keep adhesion force reduced was not developed. Hence, micro manipulation was not atomized.

Considering the above, we propose a novel method for checking adhesion force by vision. we also develop an automatic micro manipulation system. The oscillation of endeffector can not be perfectly caught by camera. Instead, the blur of the oscillation appears in the captured image. The amount of the blur is associated with the amplitude of the oscillation. Then, we develop a method to estimate the amplitude of oscillation by the blur. Subsequently, we focus that lower mode frequencies have very large amplitudes comparing with higher mode frequencies or no-resonance frequencies. Utilizing this findings, we develop a method for detecting lower mode frequencies by a blur. The adhesion state can be detected by checking whether or not lower mode frequencies are excited. Then, based on this findings and the method for detecting lower mode frequencies, we propose the method for checking adhesion state by vision. This method can be applied to any areas in the captured image and used all the time during manipulation. Its computational load is low. Since vision sensor is usually used in micro manipulation and 


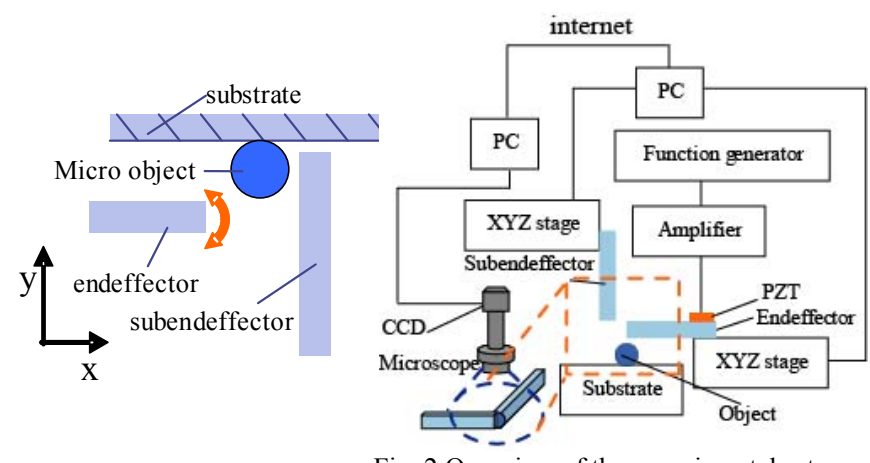

Fig. 1 Target System

Fig. 2 Overview of the experimental setup any other sensors are not needed, the total system is very simple and low cost. Applying this method to micro manipulation, we develop an automatic control system for micro manipulation.

\section{TARGET SYSTEM}

Fig. 1 shows the target system. we consider to pick and place an micro object on the substrate by endeffector and subendeffector. For the simplicity, we assume that: (1) the manipulation is done in a planner space and a gravity force doesn't work, (2) the object is a sphere, (3) the endeffector, the subendeffector, and the substrate are made of a same material, (4) the endeffector, the subendeffector, and the substrate are grounded for preventing an extra charge at the initial state. Fig. 2 shows the experimental set up. This system consists of the manipulation system, the image-capturing system, and the finger-oscillating system. The manipulation system consists of the endeffector, the subendeffector, the substrate, and the object. The endeffector and the subendeffector are cantilever beams made of copper in size of $3 \times 40 \times 0.3[\mathrm{~mm}] .5[\mathrm{~mm}]$ from the edge is used for clamping. The beams are rolled coppers, and any surface treatments such as grinding are not conducted. Young's modulus of cupper is $1.02 \times 10^{11}\left[\mathrm{~N} / \mathrm{m}^{2}\right]$, Poisson's ratio is 0.35 , and density is $8900\left[\mathrm{~kg} / \mathrm{m}^{3}\right]$. On the endeffector the piezocell (Fuji ceramics, Z0.2T50x $50 \times 50 \mathrm{~S}-\mathrm{W}$ C6) of $5 \times 3 \times 0.3[\mathrm{~mm}]$ is bonded at the position of $1[\mathrm{~mm}]$ from the clamped end for oscillating the endeffector. The surface of substrate is a cupper cut bonded on stainless board. The endeffector and the subendeffector are attached on XYZ stage (Surugaseiki, PMZG413) which can be controlled by PC. Note that the orientation of the endeffector can be slightly adjusted at the clamping area. The object is a glass sphere (Union, unibeads) with a radius of $50\left[10^{-6} \mathrm{~m}\right]$. Young's modulus of glass is $7.05 \times 10^{10}\left[\mathrm{~N} / \mathrm{m}^{2}\right]$, Poisson's ratio is 0.17 , and density is $2500\left[\mathrm{~kg} / \mathrm{m}^{3}\right]$. The image-capturing system consists of Video-microscope (Surugaseiki, VMU-V) with objective lens (Mitsutoyo S72M-5), CCD camera (Lumenera, LU135), and PC. The overview of the manipulation is captured by the CCD camera through the microscope and sent to PC. We use maximum illumination of light source (Schott MegaLight100-ROHS) whose maximum illumination is $24000[\mathrm{Lx}]$ at the $100[\mathrm{~mm}]$ from the tip of the lighting system.

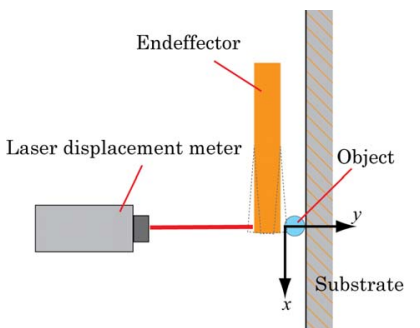

Fig. 3 Experimental set up for checking adhesion state by laser displacement meter

The endeffector is oscillated by oscillating the piezocell by a function generator (NF, DF1906) through a power amplifier. the power amplifier is handmade circuit and its amplification ratio is set to 3.2 .

\section{REDUCING ADHESION FORCE BY OSCILLATION}

Here, we introduce the method[10] for reducing adhesion force by oscillation. In a micro range, due to the scale effect, adhesion causes and makes manipulation hard. In the target system, the effect of capillary force among adhesion forces is largest. Here, we reduce such kind of adhesion force by oscillation. When the oscillated endeffector is brought close to the object on the substrate and contacts with it, the adhesion force between the object and the endeffector becomes smaller than the adhesion force between the object and the subendeffector. Then, the endeffector can be easily removed from the object while the object adheres to the substrate. However, this method can not be always available. When the amount/force of pushing the object by the endeffector is large, the effect of the oscillation decreases. Then, the adhesion force is not reduced enough, and the object sometimes adheres to the endeffector. Therefore, adhesion state has to be checked. Here, we show the experiment for the check. Fig. 3 shows the experimental set up constructed by removing the subendeffector and adding the laser displacement meter (Sony, VL10) in the system shown in Fig. 2. $y$ denotes the distance between the object and the endeffector. We move the oscillated endeffector along $y$ positive direction in Fig. 3 with the step of $1[\mu \mathrm{m}]$ until $y$ becomes $8[\mu \mathrm{m}]$. The endeffector contacts with the object at $y=0[\mu \mathrm{m}]$. We measure the oscillation of the endeffector by the laser displacement meter. The input signal for the oscillation is sine curve whose amplitude is $5[\mathrm{~V}]$, and whose frequency is 4 th mode frequency $3.171[\mathrm{kHz}]$. Fig .4 shows the results. The left figures show the movement of the endeffector and the right figures show the results obtained by applying FFT to the movement data. $y=3[\mu \mathrm{m}]$ is the case when the adhesion force is reduced while $y=8[\mu \mathrm{m}]$ is the case when the adhesion force is not reduced. From Fig. 4, it can be seen that lower mode frequencies are excited at $y=3[\mu \mathrm{m}]$ while they are not excited at $y=8[\mu \mathrm{m}]$. It indicates that we can estimate whether or not adhesion force is reduced by checking the excitation of the lower mode frequencies. 

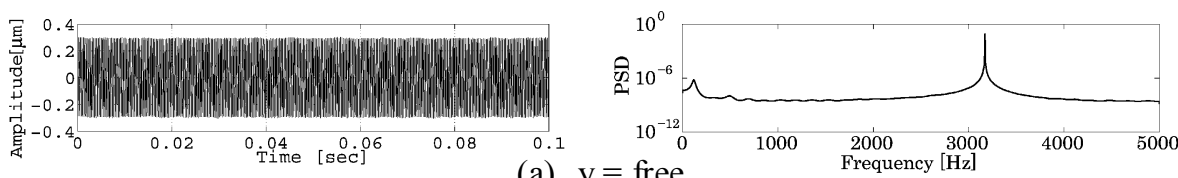

(a). $y=$ free
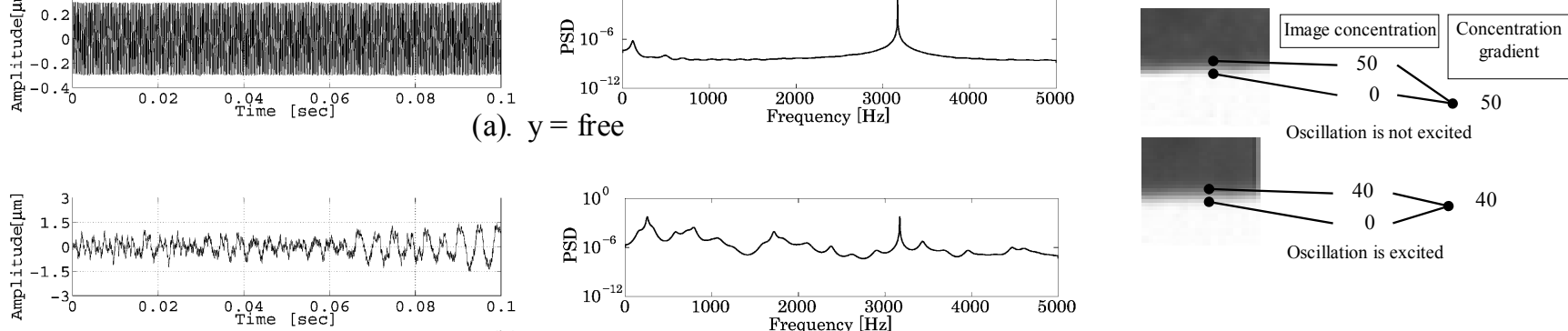

(b). $y=3$

Fig. 5 Image concentration gradient
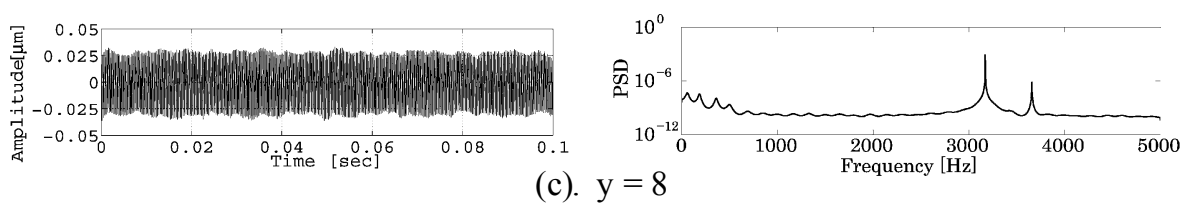

(c). $y=8$

Fig. 4 Oscillation of endeffector measured by laser displacement meter

\section{ADHESION STATE ESTIMATION BY VISION}

\section{A. Amplitude estimation by vision}

In this paper, we develop a method for detecting lower mode frequencies by vision in order to automatically control to keep adhesion force reduced. For the purpose, we firstly develop a method for estimating amplitude of oscillation using only vision information.

Oscillation is too fast to be perfectly caught by camera. However, a blur resulted from the oscillation appears in the captured image. Then, we try to estimate the amplitude of the oscillation using the blur. When oscillation is not excited, the image concentration gradient (see Fig. 5) around the edge of the endeffector is large. On the other hand, when oscillation is excited, it is small due to a blur. Using the concentration gradient, we estimate the amplitude of the oscillation. We call the estimated amplitude AIV (amplitude indicating value). The procedure for deriving AIV is shown in Fig. 6. First, the captured image is grey-scaled. Next, it is smoothed and binarized. Previously, we prepare the tip area image of the endeffector as a template image. By template matching technique[12] which find the part in the binarized image which matches the template image, we detect the position of endeffector. On the other hand, we search suitable points for tracking, $\boldsymbol{p}$, around the edge of the endeffector in the firstly grey-scaled image. Here, we pick up $\boldsymbol{p}$ 's located around the upper side (area of 10 pixel (about $10 \mu \mathrm{m}$ ) from the upper edge), and let $\mathcal{P}_{\mathrm{u}}$ be a set of the picked up points (see Fig. 7). Similarly, we pick up $\boldsymbol{p}$ 's located around the bottom side and let $\mathcal{P}_{\mathrm{b}}$ be a set of the picked up points. Also, let $\boldsymbol{p}_{\mathbf{k}-\mathbf{i}}$ be $i$ th $\boldsymbol{p}$ contained in $\mathcal{P}_{\mathrm{k}}(k \in\{u, b\})$, let $n$ be the number of $\boldsymbol{p}_{u-i} \in \mathcal{P}_{u}$, and let $m$ be the number of $\boldsymbol{p}_{b-i} \in \mathcal{P}_{b}$. We calculate $l_{\boldsymbol{k}-\boldsymbol{i}}$ which is the length between $\boldsymbol{p}_{k-i}$ and its nearest side/edge. Then, we derive the maximum value of $l_{k-i}$ :

$l_{\max }=\max _{i, k} l_{k-i}$

Using $l_{\max }$, we define the following region $X_{\mathrm{k}-\mathrm{i}}$ for $\boldsymbol{p}_{k-i}$ :

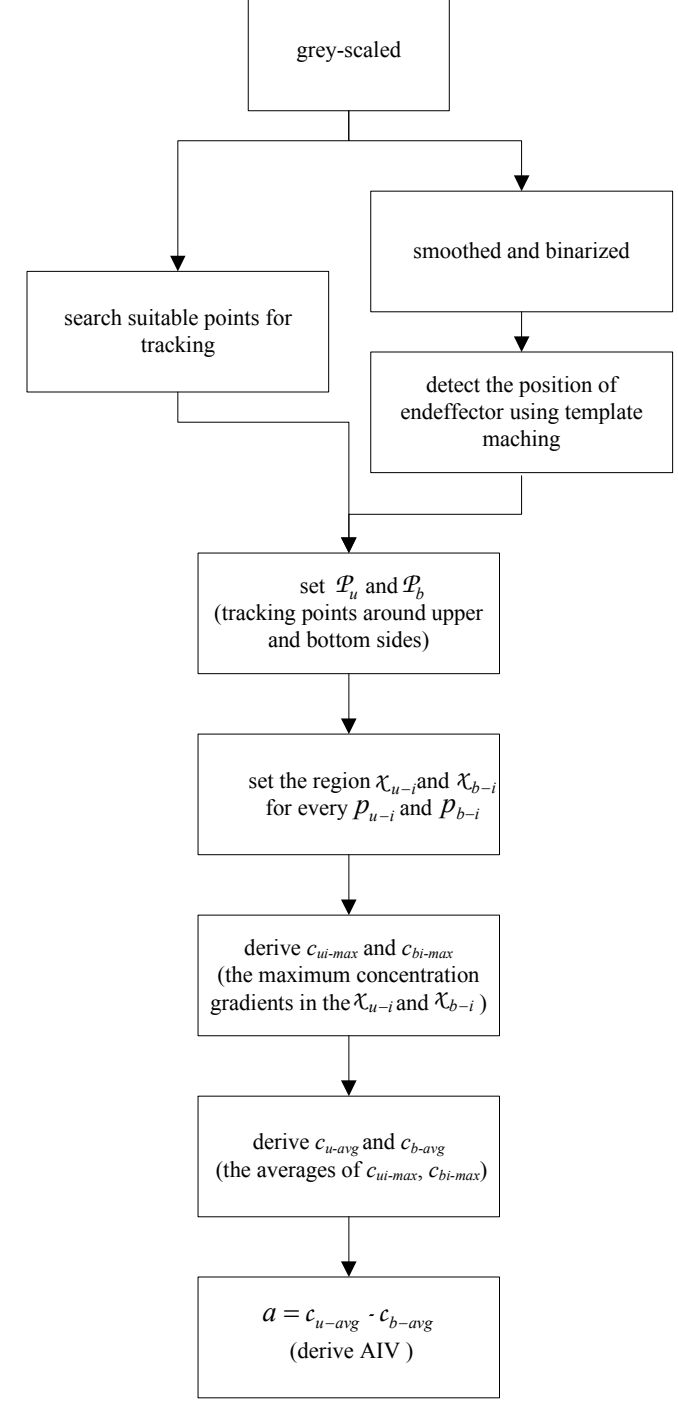

Fig. 6 Flowchart for deriving AIV (amplitude indicating value)

$x_{k-i}=\left\{(x, y) \mid \begin{array}{c}p_{k-i x}-0.6^{*} 1_{\max } \leq x \leq p_{k-i x}+0.6 * 1_{\max } \\ p_{k-i y}-1.4^{*} 1_{\max } \leq y \leq p_{k-i y}+1.4^{*} 1_{\max }\end{array}\right\}$

where $p_{k-i x}$ and $p_{k-i y}$ are, respectively, $x$ and $y$ components of $\boldsymbol{p}_{k-i}$. Here, 0.6 and 1.4 is set by trial and error so that the 


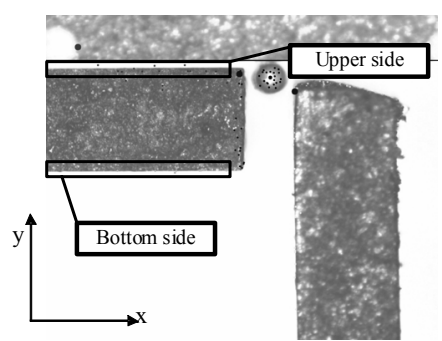

Fig. 7 Upper and bottom sides of the endeffector

nearest side/edge can be contained in $X_{k-i}$ and the variation of the maximum image concentration gradient in the $x_{\mathrm{k}-\mathrm{i}}$ can be small. Let $c_{k i-m a x}$ be the maximum concentration gradient in the $X_{\mathrm{k}-\mathrm{i}}$. We compute $c_{k-\text { max }}$ for every $\boldsymbol{p}_{k-i}\left(X_{\mathrm{k}-\mathrm{i}}\right)$ and derive its average value $c_{k \text {-avg }}$ :

$c_{u-a v g}=\left(\sum_{i=1}^{n} c_{u i-\max }\right) / n$

$c_{b-a v g}=\left(\sum_{i=1}^{m} c_{b i-\max }\right) / m$

From (3), we define the amplitude indicating value (AIV) $a$ as follows:

$a=c_{u-a v g}-c_{b-a v g}$.

Here, we confirm the efficiency of AIV by experiment. We oscillate the endeffector by oscillating PZT. The input voltage for PZT is square wave whose peak to peak is from 0 to $0-10[\mathrm{~V}]$, whose duty ratio is $50[\%]$, and whose frequency is 1 st mode frequency $0.18[\mathrm{kHz}]$. AIV is computed by the program written by $\mathrm{C}++$ language using OPEN CV library [12].

Fig. 8 shows the result. The horizontal axis expresses the input peak voltage, and the vertical axis expresses the computed AIV. Note that the input peak voltage indicates amplitude of oscillation (since the voltage is proportional to the amplitude). By applying regression analysis, the relation is expressed by $v=85 a^{-0.27}$ where $v$ denotes the input peak voltage. From the result, it can be seen that the amplitude of oscillation can be estimated by AIV.

\section{B. Discrimination between higher and lower mode frequencies by AIV}

When bringing the oscillated endeffector with higher mode frequency close to the object on the substrate and lower mode frequencies are excited, the adhesion force between the endeffector and the object is reduced. Then, if discriminating between higher and lower mode frequencies by AIV, we can detect the adhesion state by AIV. Here, we investigate whether or not higher and lower mode frequencies can be discriminated by AIV by experiment.

The input voltage for PZT is square wave whose peak to peak is from 0 to $24[\mathrm{~V}]$, whose duty ratio is $50[\%]$, and whose frequency is 1 st -4 th mode frequency. Here, we select from 1st to 4th mode frequencies, considering 4th mode frequency is used in our previous paper[10]. For the comparison, we also select non-resonance frequency of 2

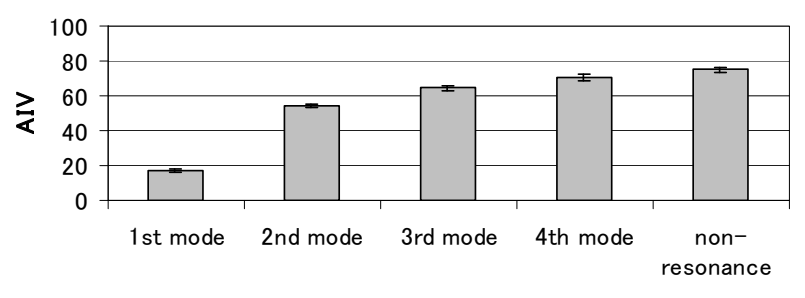

Fig. 9 AIV for 1-4th mode frequency

$[\mathrm{kHz}]$.

Fig. 9 shows the results. From Fig. 9, it can be seen that the higher the frequency mode is, the larger AIV is. It is thought to come from that the higher frequency mode is, the smaller the amplitude is. The difference between AIV's for 1st mode and the other modes (including non-resonance frequency) is very large, and then the 1 st mode oscillation can be easily detected. The 2 nd mode can also be discriminated from the other higher mode oscillations by checking the difference of AIV. On the other hand, the discriminations between 3rd and 4th modes and between 4th mode and non-resonance frequencies are not easy, although they are possible.

\section{Detection of adhesion state by AIV}

In this subsection, base on the previous subsection results, we investigate whether adhesion state can be detected by AIV.

We take the following way (see Fig. 10). First, we bring the oscillated endeffector close to the object and contact the endeffector with the object. Next, we move the endeffector in the left and right directions of this page. If adhesion force is reduced, the endeffector slides on the object while the object is at stationary state. If can not, the object rotates. In the case when the endeffector slides on the object, we increase the amount of pushing the object, and move the endeffector in the left and right directions again. This procedure is repeated until the object rotates. The input voltage for the oscillation is square wave whose peak to peak is from 0 to 24 [V], whose duty ratio is $50[\%]$, and whose frequency is 4 th mode frequency $3.456[\mathrm{kHz}]$. The experience was done 5 times.

The results are shown in Fig. 11. Free means the endeffector is oscillated without contacting with the object. Note that in this experiment, the value of AIV when adhesion force is reduced changes with the change of the amount of pushing the object. Then, AIV in that case is shown by range. From Fig. 11, it can be seen that AIV when adhesion force is reduced is smaller than or equal to AIV for free state. The maximum difference is about 20. It is the reason why the lower mode frequencies (than the frequency of the input oscillation) are excited. On the other hand, AIV when adhesion force is not reduced is larger than AIV for free state. It is the reason why if the amount of pushing the object is large, the energy of oscillation decreases and then the amplitude of the oscillation becomes smaller than that in the free state (refer to Fig. 4).

Note that AIV for 4th mode in Fig. 9 is different from AIV 


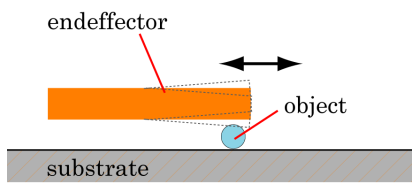

Fig. 10 Overview of the experiment for checking whether adhesion state can be detected by AIV

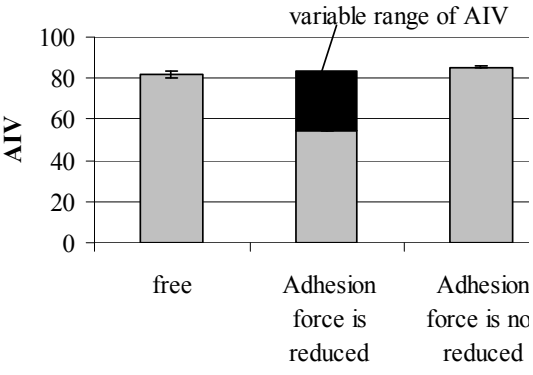

Fig. 11 AIV's when adhesion force is reduced and not reduced (AIV is shown by range because AIV when adhesion force is reduced changes with the change of amount of pushing the object) for free state in Fig. 11. It is due to the large difference of the endeffector position. The illumination or light intensity differs from place to place. Then, if the endeffector position differs largely, AIV also differs. Therefore, in order to check adhesion state, we use the difference between AIV when the endeffector is freely oscillated around the target point and AIV when the endeffector contacts with the object. Note also that there is the case when AIV when adhesion force is reduced is almost same as AIV for free state. It is thought that the lower mode frequencies are excited but their amplitude is small, and then AIV is large. In such a case, it is difficult to detect adhesion state. However, a precious control for the amount of pushing the object is not need in the target operation. Therefore, we only have to control endeffector so that the difference between AIV's for free and contacting cases can be included in the appropriately defined range. Then, we can keep adhesion force reduced, while pushing the object with enough large force.

\section{AUtOMATIC MiCRO MANIPULATION SYSTEM}

Using the developed method for detecting the adhesion state by vision, we develop a system which automatically pick and place a micro object. We use the experimental set up shown in Fig. 1 and Fig. 2.

We show the procedure for picking operation in Fig. 12 (refer to the real movement shown in Fig. 14). First, using template matching technique, we find the tip positions of the endeffector and the subendeffector, and the geometric center of the object. The endeffector is oscillated. Using the position information, we pinch the object between them. Next, we remove the object from the substrate by moving (controlling) the endeffector and the subendeffector. Subsequently, we remove the endeffector from the object. In this case, the oscillation of the endeffector can reduce the adhesion force between the endeffector and the object (see [10] for detail). We check the difference of AIV and judge the control of endeffector (adhesion force) is not needed, and then the checking and controlling procedure is not included in the flowchart shown in Fig. 12. Next, placing operation shown in Fig. 13 is done. First, using template matching technique, we find the tip positions of the endeffector and the

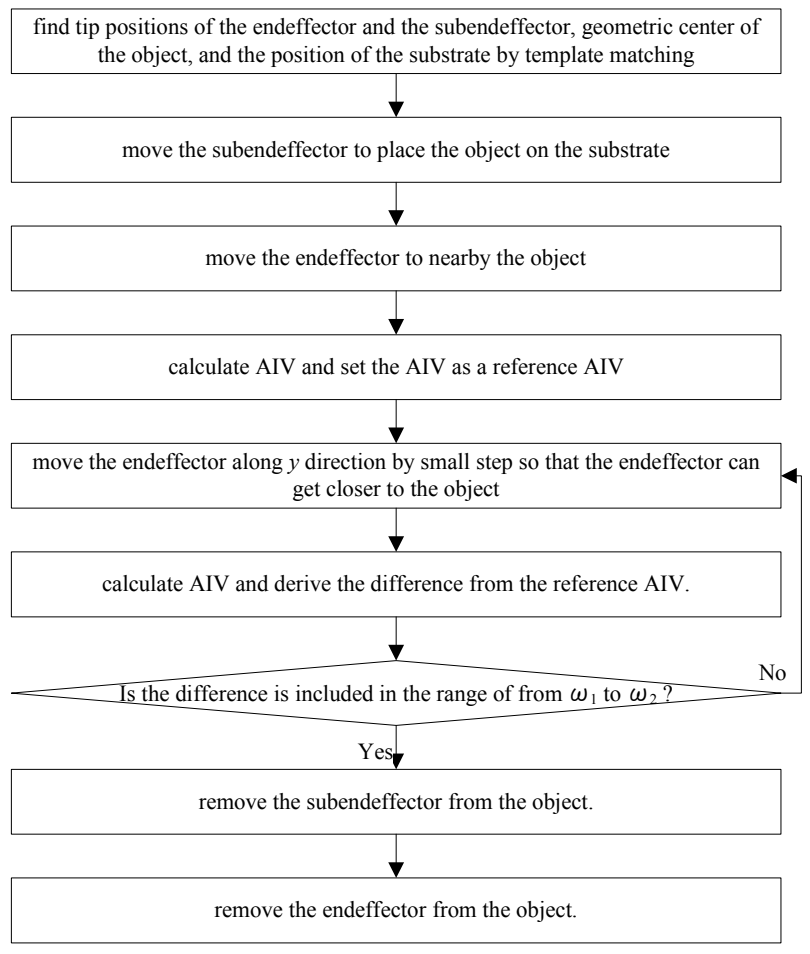

Fig. 13 Flowchart for placing operation

subendeffector, the geometric center of the object, and the position of the substrate. Using the information, we place the object on the substrate by moving (controlling) the subendeffector. Next, we move the endeffector to nearby the object so that the endeffector can contact with the object and push it if moving the endeffector along $y$ direction (refer to $y$ direction in Fig. 1). The endeffector is oscillated. Here, we calculate AIV and set it as the reference AIV. Next, we move the endeffector along $y$ direction by small step so that the endeffector can get closer to the object. We calculate AIV and derive the difference from the reference AIV. If the difference is not included in the range of from $\omega_{1}$ to $\omega_{2}$, we move the endeffector along $y$ direction by small step, and calculate AIV and the difference from the reference AIV again. If the difference is included in the range, we judge that the endeffector push the object by enough large force, reducing the adhesion force. In this case, we stop moving the 


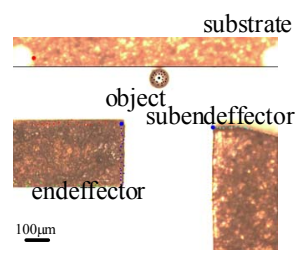

(1)

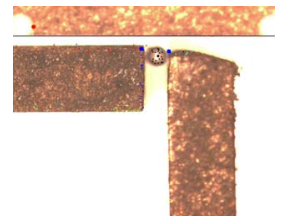

(3)

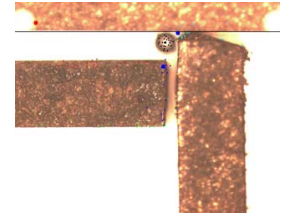

(5)

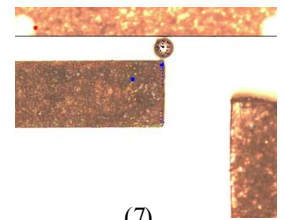

(7)

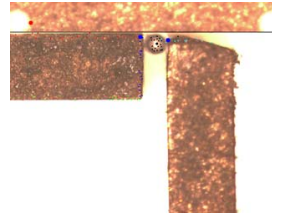

(2)

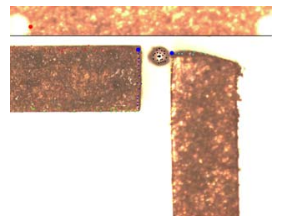

(4)

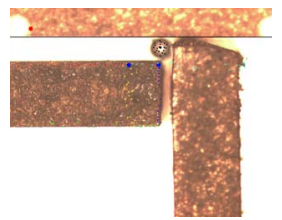

(6)

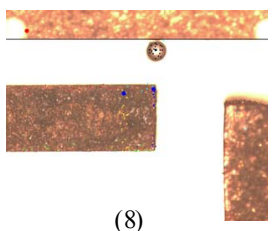

(8)
Fig. 14 Overview of the experiment for automatic pick and place micro operation

endeffector and remove the subendeffector from the object. Subsequently, we remove the endeffector from the object, and finish the operation.

The input voltage for PZT is square wave whose peak to peak is from 0 to $24[\mathrm{~V}]$, whose duty ratio is $50[\%]$, and whose frequency is 4 th mode frequency $3.456[\mathrm{kHz}]$. The threshold value $\omega_{1}$ and $\omega_{2}$ are, respectively, set to 5 and 10 by try and error so that the difference from the reference AIV can be detected, while the endeffector can apply enough large pushing force to the object.

The outline of the result is shown in Fig. 14. The number denotes the order of the time line.

We did this operation 5 times, and all operations were successfully done. These results indicates the validity of our approach.

\section{CONCLUSION}

In our previous paper [10], we proposed a method for reducing adhesion force by oscillation. However, the following problems have not been still resolved: (1) Since adhesion state was checked by analyzing the data obtained by laser displacement meter, this method is available in only limited situations. (2) Automatic checking system for adhesion state was not developed. (3) Automatic micro manipulation system was not developed. Considering the above, in this paper, we have proposed a method to automatically check adhesion state, using only vision information. Firstly, we have developed a method to estimate the amplitude of the oscillation, using the blur resulted from the oscillation. We call the estimated amplitude AIV (amplitude indicating value). Using this method, we have showed lower mode oscillations can be discriminated from higher mode oscillations. Then, we have developed a method for checking adhesion state by AIV. Based on the checking method, we have developed a automatic micro manipulation system for pick and place operation. The validity of our method has been shown by experiments. The target manipulation doesn't need precious control of (adhesion) force. Then, the proposed method is available. If applying this method to another manipulation which needs more precious force control, we need to develop a more robust method including illumination (noise) effect. It's our future work.

\section{REFERENCES}

[1] R. S. Fearing, "Survey of sticking effects for micro-parts", Proc. of the IEEE/RSJ Int. Conf. on Intelligent Robots and Systems, 1995, pp. 212-217.

[2] F. Arai, D. Andou, and T. Fukuda, "Micro Manipulation Based on Physical Phenomena in Micro World (1st Report, The Reduction Method of Van Der Waals Force)", Trans. of the Japan Society of Mech. Eng., Series C, vol.62, no.603, 1996, pp. 4286-4293 (in Japanese).

[3] F. Arai, D. Andou, Y. Nonoda, T. Fukuda, "Micro Manipulation Based on Physical Phenomena in Micro World(Principle and Prototype Experiments of Adhesion type Micro Endeffector)", Trans. of the Japan society of Mech. Eng., series C, vol.62, no.604, 1996, pp.4630-4635.

[4] W. Zesch, M. Brunner, and A.Weber, "Vacuum tool for handling micro objects with a nano-robot", Proc. of the IEEE Int. Con. on Robotics and Automation, 1997, pp.1761-1766.

[5] Y. Rollot, S. Regnier, and J. Guinot, "Dynamical model for the micromanipulation by adhesion : Experimental validations for determined conditions", J. of Micromechatronics, vol.1, no.4, 2002, pp.273-297.

[6] D. S. Haliyo, Y. Rollot, and S. Regnier, "Manipulation of micro-objects using adhesion forces and dynamical effects", Proc. of the IEEE Int. Conf. on Robotics and Automation, 2002, pp.1949-1954.

[7] D. S. Haliyo and S. Regnier, "Advanced applications using $\ddot{u} \mathrm{mad}$, the adhesion based dynamic micro-manipulatior", Proc. of the IEEE/ASME Int. Conf. on Advanced Intelligent Mechatronics, 2003, pp. 880-885.

[8] S. Saito, H. Himeno, and K. Takahashi, "Electrostatic detachment of an adhering particle from a micromanipulated probe", J. of Applied Physics, vol. 93, no. 4, 2003, pp. 2219-2224.

[9] S. Saito, H. T. Miyazaki, T. Sato, K. Takahashi, and T. Onzawa, "Analysis of micro-object operation based on the dynamics considering the adhesion under an sem", Proc. of the IEEE/RSJ Int. Conf. on Intelligent Robots and Systems, 2001, pp. 1349-1357.

[10] T. Watanabe, M. Iwasaki, H. Matsumura, Z. Jiang, "Adhesion Forces Relaxation by Oscillation and Its Application to Micro Manipulation", Trans. of the Japan society of Mech. Eng., series C, Vol.74, No.737, 2008, pp.23-30(in Japanese).

[11] T. Watanabe and S. Fukui, "A method for controlling tactile sensation of surface roughnessusing ultrasonic vibration", Proc. of the IEEE Int. Con. on Robotics and Automation, 1995, pp.1134-1139.

[12] OpenCV Reference manual, Available: http://opencv.jp/opencv/document/

[13] B. D. Lucas, T. Kanade, "An lteretive Image Registration Technique with an Application to Stereo Vision", Proc. 7th Int. Conf. on Artificial Intelligence, 1981, pp.674-679. 